\title{
Evaluación del riesgo ambiental terrestre de dos gasolinas ecológicas de 90 octanos en la lombriz de tierra Eisenia Fetida (Savigny, 1826)
}

Evaluation of the terrestrial environmental risk of two ecological 90
octane gasolines in the earthworm Eisenia Fetida (Savigny, 1826)
Recibido: mayo 05 de 2020 | Revisado: junio 12 de 2020 | Aceptado: julio 11 de 2020

\author{
Susana Isabel Avalos-Ruiz \\ José IANNACONE ${ }^{\mathrm{I}, 2,3}$
}

1 Laboratorio de Ingeniería Ambiental. Carrera de Ingeniería Ambiental. Universidad Científica del Sur (UCSUR), Lima, Perú.

2 Grupo de Investigación en Sostenibilidad Ambiental (GISA), Escuela Universitaria de Posgrado (EUPG), Laboratorio de Ecología y Biodiversidad Animal (LEBA). Facultad de Ciencias Naturales y Matemática (FCCNM). Universidad Nacional Federico Villarreal (UNFV), Lima, Perú.

3 Laboratorio de Parasitología. Facultad de Ciencias Biológicas. Universidad Ricardo Palma. Lima, Perú. Autor para correspondencia E-mail: jiannaconeo@unfv. edu.pe

\begin{abstract}
Resumen
Se evaluó el riesgo ambiental terrestre de dos gasolinas ecológicas de 90 octanos sobre la lombriz de tierra Eisenia fetida (Savigny, 1826). El parámetro de toxicidad letal evaluado fue la mortalidad, y los efectos subletales fueron seis: movilidad, oscurecimiento, adelgazamiento, estrangulación, fraccionamiento y peso corporal a los 7 y 14 días de exposición. Los organismos fueron expuestos a cinco concentraciones de gasolinas ecológicas de 90 octanos expresadas en base al xileno: 49,5 ug. $\mathrm{kg}^{-1}, 148,5 \mathrm{ug} \cdot \mathrm{kg}^{-1}, 346,5 \mathrm{ug} \cdot \mathrm{kg}^{-1}, 841,5 \mathrm{ug} \cdot \mathrm{kg}^{-1}$ y $2079 \mathrm{ug} \cdot \mathrm{kg}^{-1}$. Las gasolinas ecológicas de 90 octanos (Petroperú y Repsol) en el suelo no presentaron efectos de toxicidad letal (concentración letal media, $\mathrm{CL}_{50}$ ) en E. fetida. Los efectos subletales fueron inmovilidad, oscurecimiento y adelgazamiento. Se consideró como criterio de comparación el Estándar de Calidad Ambiental para suelos (ECA) para el Perú expresado en xileno $\left(11 \mathrm{mg} \cdot \mathrm{kg}^{-1}\right)$. En base a este ECA, los parámetros más sensibles para la gasolina-Petroperú que ocasionaron riesgo en la lombriz de tierra fueron inmovilidad, oscurecimiento y adelgazamiento en relación a la Concentración en la que no se observa efecto (NOEC). Para la gasolina - Repsol, los mismos parámetros mostraron riesgo en función al NOEC; sin embargo, el peso húmedo presentó un riesgo significativo en la lombriz al igual en base a la concentración efectiva media $\left(\mathrm{CE}_{50}\right)$. La gasolina-Repsol presentó una mayor toxicidad para la lombriz de tierra que la gasolina-Petroperú, y el riesgo ambiental fue mayor para las respuestas subletales.
\end{abstract}

Palabras clave: bioensayos, $\mathrm{CE}_{50}, \mathrm{CL}_{50}$, ecotoxicología, Eisenia fetida, gasolina, NOEC, toxicidad letal, toxicidad subletal

\begin{abstract}
The terrestrial environmental risk of two ecological 90 octane gasoline was evaluated on the earthworm Eisenia fetida (Savigny, 1826). The lethal toxicity parameter evaluated was mortality, and the sublethal effects were six: mobility, darkening, thinning, strangulation, fractionation, and body weight at 7 and 14 days of exposure. The organisms were exposed to five concentrations of ecological gasoline of 90 octane expressed on the basis of xylene: $49.5 \mathrm{ug} \cdot \mathrm{kg}^{-1}, 148.5 \mathrm{ug} \cdot \mathrm{kg}^{-1}, 346.5 \mathrm{ug} \cdot \mathrm{kg}^{-1}, 841.5 \mathrm{ug} \cdot \mathrm{kg}^{-1}$ and $2079 \mathrm{ug} \cdot \mathrm{kg}^{-1}$. The ecological 90 octane gasoline (Petro Peru and Repsol) in the soil did not show lethal toxicity effects (medium lethal concentration, $\left.\mathrm{LC}_{50}\right)$ in E. fetida. The sublethal effects were immobility, darkening, and thinning. The Environmental Quality Standard for soils (ECA) for Peru expressed in xylene $\left(11 \mathrm{mg} \cdot \mathrm{kg}^{-1}\right)$ was considered as a comparison criterion.
\end{abstract}

(C) Los autores. Este artículo es publicado por la Revista Campus de la Facultad de Ingeniería y Arquitectura de la Universidad de San Martín de Porres. Este artículo se distribuye en los términos de la Licencia Creative Commons Atribución No-comercial - Compartir-Igual 4.0 Internacional (https://creativecommons.org/licenses/ CC-BY), que permite el uso no comercial, distribución y reproducción en cualquier medio siempre que la obra original sea debidamente citada. Para uso comercial contactar a: revistacampus@usmp.pe. 
Based on this ECA, the most sensitive parameters for gasoline-Petroperu that caused risk in the earthworm were immobility, darkening and thinning in relation to the Concentration in which no effect is observed (NOEC). For gasoline-Repsol, the same parameters showed risk based on NOEC; however, the wet weight presented a significant risk in the earthworm as well as based on the mean effective concentration $\left(\mathrm{EC}_{50}\right)$. Gasoline-Repsol presented a higher toxicity for the earthworm than gasoline-Petroperu, and the environmental risk was greater for the sublethal responses.

Key words: $\mathrm{CE}_{50}, \mathrm{CL}_{50}$, ecotoxicology, Eisenia fetida, gasoline, LOC, lethal toxicity, NOEC, sublethal toxicity

\section{Introducción}

La industria de hidrocarburos es una fuente indispensable en el marco productivo del Perú (Bravo, 2007). Dentro de la gama de los hidrocarburos, las gasolinas son las más comerciales debido al incremento y demanda del parque automotor y según sea el aditivo agregado generan el octanaje de la gasolina (Murrugarra, 2009). La gasolina de 90 octanos o comúnmente llamada gasolina ecológica (Repsol, 2007) está constituida por una mezcla de hidrocarburos saturados, olefinas, naftenos y aromáticos (Petroperú, 2013; Pinedo et al., 2013; Abdollahinejad et al., 2020).

Los derrames incontrolados de combustibles en estaciones de servicio y en plantas industriales han generado un deterioro significativo de los ecosistemas naturales tanto contaminación del suelo como de las aguas subterráneas (Flores $\&$ Bitteri, 2000). El derrame de gasolina es considerado como uno de los accidentes ambientales con mayor dificultad para su mitigación o remediación, pero a la par estos derrames se están presentando con mayor frecuencia debido al mal estado de las vías de transporte y de la disposición final (Flores et al., 2004; Lemos et al., 2020).
Desde hace algunos años atrás, el uso de organismos biológicos llamados bioindicadores en ensayos de ecotoxicidad está ampliamente documentado, por este motivo, en años recientes se han usado otras respuestas biológicas "biomarcadores" (Iannacone \& Alvariño, 2004, 2005; Iannacone et al., 2006; Iannacone et al., 2009; Khan et al., 2018; Escobar-Chávez et al., 2019; Bamgbose \& Anderson, 2020; Bautista-Medina \& Iannacone, 2020; Lemos et al., 2020). Para tal efecto, los ensayos ecotoxicológicos pueden ser empleados en diversos niveles, desde el sub-individual (evaluación de efectos moleculares y bioquímicos) hasta el nivel poblacional, comunidad y ecosistema (Kammenga et al., 2000; Iannacone \& Alvariño, 2005; da Silva Júnior et al., 2018; Khan et al., 2018; Escobar-Chávez et al., 2019; Bamgbose \& Anderson, 2020; Bautista-Medina \& Iannacone, 2020; Lemus et al., 2020).

La lombriz de tierra o la lombriz roja californiana Eisenia fetida (Savigny, 1826) (Annelida) es considerada a nivel mundial como una de las más representantes dentro la macrofauna del suelo y dentro de todas las lombrices de tierra en particular (OECD, 1984; da Silva Júnior et al., 2018). Eisenia fetida, es de gran importancia gracias a que cumple un rol ecológico primordial 
(Sanchez-Hernandez, 2006; Lemos et al., 2020), como por ejemplo causan modificaciones físicas en el suelo así como al mismo tiempo incrementa las posibilidades de desarrollo de otros animales y plantas generando un equilibrio en el ecosistema (Brown et al., 2004; Abdollahinejad et al., 2020). Adicionalmente las lombrices de tierra son capaces de acumular varios contaminantes orgánicos e inorgánicos presentes en el suelo (Morrison et al., 2000; Boyd et al., 2001; Peijnenburg, 2002; Cuevas et al., 2008; Abdollahinejad et al., 2020). La lombriz de tierra ha sido propuesta, extensivamente, en estudios ecotoxicológicos (Scott-Fordsmand \& Weeks, 2000; da Silva Júnior et al., 2018; Khan et al., 2018; Bamgbose \& Anderson, 2020).

La presente investigación evidencia que la lombriz de tierra es un modelo biológico estándar para identificar impactos ecosistémicos potenciales por derrame de hidrocarburos al suelo, lo que será de mucha utilidad en el Perú (Abdollahinejad et al., 2020), debido a que actualmente no se cuenta con un registro detallado sobre el efecto ecotoxicológico que generan los derivados de hidrocarburos-gasolinas de mayor circulación, distribución y venta en el Perú (da Silva Júnior et al., 2018; Khan et al., 2018). Adicionalmente, esta investigación sirve de modelo para mejorar la normativa para un mejor cuidado del medio ambiente en el sector público, mientras que en el sector privado ayudará a implementar protocolos de monitoreo ambiental. El objetivo de la presente investigación fue determinar el riesgo ambiental terrestre de dos gasolinas ecológicas de 90 octanos en la lombriz de tierra.

\section{Materiales y Métodos}

\section{Fundamento del protocolo}

Se seleccionó el protocolo del ensayo de toxicidad letal estático (Cuevas et al., 2008) y el efecto tóxico subletal en lombriz de tierra E. fetida a nivel laboratorio y descrito en la guía 207 de la OECD para evaluación de sustancias (OECD, 1984).

\section{Obtención y selección del material biológico}

Se escogió a $E$. fetida por ser una especie indicadora de calidad ambiental del suelo (Cuevas et al., 2008). Se seleccionaron individuos de similar tamaño y con presencia del anillo clitelar desarrollado y visible, el cual es indicativo de su capacidad reproductiva (OECD, 1984). Las lombrices adultas se seleccionaron de un mismo cultivo (con el fin de evitar variaciones en los resultados), con un peso corporal en el rango $100-300 \mathrm{mg}$ (promedio $=192 ; \mathrm{n}=280$ ).

\section{Preparación de sustrato artificial}

La preparación del suelo artificial consistió en la mezcla de $10 \%$ de musgo de turba (materia orgánica), 20\% de arcilla, y $70 \%$ de arena industrial (partículas entre 50-200 $\mu \mathrm{m}$, previamente tamizada. Del mismo modo, se le agregó la cantidad de agua necesaria para obtener una humedad aproximada del 35\%. La preparación se mezcló hasta conseguir homogeneidad (OECD, 1984). En la Tabla 1, se detallan los valores más representativos de caracterización de la muestra de suelo usados en los bioensayos con E. fetida. 
Tabla 1

Análisis de caracterización del suelo usado en los bioensayos con Eisenia fetida. AF = Arena Franca

\begin{tabular}{lll}
\hline Materia Orgánica $(\%)$ & 5,7 & Método de Walkley \& Black \\
$\mathrm{pH}(1: 1)$ & 6,7 & Medida del potenciómetro de la suspensión suelo \\
$\mathrm{CE}\left(\mathrm{dS} \cdot \mathrm{m}^{-1}\right)$ & 2,91 & Medida en extracto acuoso relación- suelo: agua \\
$\mathrm{CaCO} 3(\%)$ & 1,1 & Método Gaso-volumétrico \\
$\mathrm{CIC}\left(\mathrm{meq} \cdot 100 \mathrm{~g}^{-1}\right)$ & 9,6 & Método de saturación con acetato de amonio \\
Clase Textual & AF & Método del hidrómetro \\
\hline
\end{tabular}

En la Tabla 2, se muestra que predominó la fracción arena por el método del hidrómetro; por lo tanto, la

Tabla 2

Análisis de granulometría del suelo usado en los bioensayos con Eisenia fetida

\begin{tabular}{ll}
\hline \% Arena muy gruesa $(2,00-1,00 \mathrm{~mm})$ & 0,46 \\
\% Arena gruesa $(1,00-0,50 \mathrm{~mm})$ & 5,16 \\
\% Arena media $(0,50-0,25 \mathrm{~mm})$ & 26,8 \\
\% Arena fina $(0,25-0,10 \mathrm{~mm})$ & 39,92 \\
\% Arena muy fina $(0,10-0,05 \mathrm{~mm})$ & 11,04 \\
$\%$ Limo & 8,98 \\
\% Arcilla & 7,64 \\
\hline
\end{tabular}

\section{Aclimatación de lombrices}

Para llevar a cabo la aclimatación de las lombrices se realizaron dos actividades: (1) previo a la aclimatación, se les dieron todas las condiciones necesarias, su alimentación consistió de tomates "cherry" descompuestos del huerto de la Universidad Científica del Sur (UCSUR), Lima, Perú; (2) posteriormente, las lombrices seleccionadas fueron aclimatadas en suelos de similares condiciones a lo largo de una semana y no recibiendo alimento durante el bioensayo (OECD, 1984).

\section{Los ensayos de aclimatación} fueron considerados validos cuando la mortalidad de E. fetida no sobrepasó el 10\% (Iannacone \& Alvariño, 2004). La cantidad de agua de riego suministrada cada dos a tres días durante ensayo fue de 50 a $75 \mathrm{~mL}$, la humedad en suelo se mantuvo en el rango óptimo establecido de 40-80\%, considerando la respuesta aceptable de los bioensayos (Alonzo \& Chicas, 2013).

\section{Sustancias químicas}

Se utilizó gasolinas ecológicas de 90 octanos de dos proveedores: Repsol ( $\mathrm{pH}$ aproximado de 6 y densidad aproximada de $0,71-0,73)$ y Petroperú (pH aproximado de 5,5 y densidad aproximada de 0,75). Para ambos casos se asumió una densidad de $0,75 \mathrm{~g} \cdot \mathrm{cm}^{-3} \mathrm{y}$ se utilizó el concentrado equivalente a un galón de gasolina a $15^{\circ} \mathrm{C}$. Para identificary seleccionar el valor de las concentraciones utilizadas en las series experimentales, se consideraron los valores registrados en la literatura científica (Iannacone \& Alayo 2002; Iannacone \& Alvariño 2004), 
tomando en cuenta que la gasolina tiene la siguiente composición: 4-8\% alcanos; $2-5 \%$ alquenos; $25-40 \%$ isoalcanos; $3-7 \%$ cicloalcanos; $1-4 \%$ ciclcloalquenos; y $20-50 \%$ aromáticos totales (ATSDR, 1995).

Las concentraciones de gasolina de 90 octanos se consideraron en función al compuesto predominante en los aromáticos totales que fue el xileno. Para determinar las concentraciones de las dos gasolinas de 90 octanos se tomó en cuenta el 6,6\% de pureza del xileno. $\mathrm{Se}$ determinó este elemento debido a que es el compuesto predominante en la composición de los aromáticos totales, y estos a su vez predominan en la composición de la gasolina. Así mismo es el elemento de mayor persistencia en suelo (ATSDR, 1995; Pinedo, 2014; NTP, 2017).

Las concentraciones en base al xileno fueron: $49,5 \mathrm{ug} \cdot \mathrm{kg}^{-1}, \quad 148,5 \mathrm{ug} \cdot \mathrm{kg}^{-1}$, $346,5 \mathrm{ug} \cdot \mathrm{kg}^{-1}, \quad 841,5 \mathrm{ug} \cdot \mathrm{kg}^{-1}$ y 2079 $\mathrm{ug} \cdot \mathrm{kg}^{-1}$. Los tratamientos fueron: cinco concentraciones y dos controles (un control de agua y un control mezcla de alcohol y agua). Adicionalmente, se realizaron cuatro repeticiones por cada tratamiento (Iannacone \& Alayo, 2002; Cuevas et al., 2008).

\section{Bioensayos}

Para realizar el montaje de los bioensayos se seleccionaron contenedores de plástico. Cada contenedor contuvo $1 \mathrm{~kg}$ de suelo artificial con cada tratamiento y 10 lombrices en cada uno, las cuales fueron previamente climatizados. Se comprobó mediante la repuesta a estímulos de tacto y escape de la luz que las lombrices estaban saludables antes de iniciar el experimento (Gómez, 2014; Cuevas et al., 2008ab; Palafox et al., 2012). El punto final considerado como respuesta letal fue la mortalidad, evaluada a través de $\mathrm{CL}_{50}$, y los puntos finales de respuesta subletal fueron siete: inmovilidad, oscurecimiento, adelgazamiento, estrangulación, fragmentación, peso seco y peso húmedo evaluado a través de $\mathrm{CE}_{50}$ (Concentración efectiva media), LOEC (Concentración más baja de efectos observables) y NOEC (Concentración de efectos no observables) para las gasolinas de Repsol y Petroperú a 7 y 14 días de exposición (OECD, 1984; Schaefer et al., 2005).

Durante la aclimatación y el desarrollo del bioensayo, se controlaron la temperatura y humedad relativa, los cuales se midieron con un termohidrómetro $\left(\right.$ Coolbox $\left.^{\oplus}\right)$. El comportamiento de la temperatura se mantuvo poco fluctuante, esto debido a que el bioensayo se realizó en el invernadero de la UCSUR, y se encontró que la temperatura más alta fue de $24,6^{\circ} \mathrm{C}$, mientras que la más baja fue de $19^{\circ} \mathrm{C}$ (promedio $=21,29 \pm 1,54^{\circ} \mathrm{C}$ ) y un rango de $19-24,6^{\circ} \mathrm{C}$. Con respecto a la humedad relativa, presentó valores estables con un promedio de $73 \% \pm 0,08$; dentro de un rango de $60-88 \%$ cada semana aproximadamente.

\section{Evaluación de Riesgo Ambiental (ERA)}

Para determinar el riesgo de la gasolina de 90 octanos, se eligieron los resultados de los ensayos de toxicidad más sensibles, o sea el valor menor de NOEC y CE (L) ${ }_{50}$ a partir de los datos obtenidos de los efectos letales y subletales en los bioensayos con E. fetida. Para establecer los valores de las concentraciones que no causan efectos sobre los organismos, se determinó el PNEC (concentración 
por debajo de la cual se estima que no se producirán efectos adversos sobre los organismos) y se calculó en base a los siguientes valores por separado. Los valores de NOEC se dividieron entre un factor de seguridad de 100 y los valores de la concentración efectiva media CE (L) ${ }_{50}$ se dividieron entre 1000 (IHCP, 2003).

Para tal efecto, se determinó la exposición al contaminante, tomando el valor del compuesto xileno indicado en el Estándar de Calidad Ambiental de Suelo de la norma peruana (Decreto Supremo $\mathrm{N}^{\circ}$ 002-2013-MINAM). Finalmente se determinó el riesgo a través de la evaluación del cociente de riesgo (CR) dividiendo la concentración de exposición (ECA suelo) entre el PNEC estimado (Delgado-Blas \& Uc-Peraza, 2015). Es preciso indicar que un cociente de riesgo inferior a un 1 representa generalmente un riesgo aceptable, mientras que un cociente superior a un nivel crítico comparativo (LOC) equivalente a 1 se considera preocupante por tratarse de una exposición muy elevada que puede representar un riesgo (Planes \& Fuchs, 2015).

\section{Análisis estadístico}

A través de un análisis de varianza (ANDEVA) en bloques completamente aleatorios se evaluó la eficacia de las concentraciones de las gasolinas entre las repeticiones $\mathrm{y}$ finalmente entre los tiempos) de exposición (7 y 14 días). Como se presentaron diferencias significativas entre las réplicas, se realizó una prueba de significancia (Diferencia Verdaderamente Significativa-DVS) mediante una prueba de Tukey (Iannacone \& Alayo, 2002). Por su parte, las CE (L) s50, se calcularon usando el programa computarizado Probit versión 1,5 de la USEPA. Para probar diferencias entre las medias de las lecturas a los 7 y 14 días de exposición se realizó una prueba t-student para muestras relacionadas y simultáneamente se corrigieron con la fórmula de Schneider-Orelli's, la misma que permite ajustar los valores a cero cuando existe mortalidad en control pero no supera el $10 \%$. Asimismo los datos obtenidos se analizaron con el paquete estadístico SPSS 20,0 para establecer si existían o no diferencias significativas. Se consideró en todos los casos un valor de $\mathrm{p} \leq 0,05$ como significativo (EPA, 1998).

\section{Resultados}

\section{Gasolina de 90 octanos-Petroperú expresada en base al xileno (ug. $\mathrm{kg}^{-1}$ )}

La Tabla 3 muestra los valores en porcentaje de los parámetros evaluados: mortalidad, inmovilidad,oscurecimiento, adelgazamiento, estrangulación, fraccionamiento y peso) sobre la lombriz de tierra E. fetida bajo el efecto de cinco concentraciones crecientes de gasolina de 90 octanos-Petroperú y expresadas en ug de xileno en $\mathrm{kg}^{-1}$ de suelo.

No se observó efecto letal en $E$. fetida en relación a la gasolina de 90 octanosPetroperú. Se determinó que el NOEC fue la concentración más alta utilizada durante el bioensayo. Se observó un incremento en el porcentaje de mortalidad a los 14 días de exposición $(\mathrm{t}=4,50$; $\mathrm{p}=0,004)$. En el parámetro inmovilidad, se observaron diferencias estadísticamente significativas en los bioensayos a los 7 y 14 días de exposición. Se determinó que el LOEC fue de $49,5 \mathrm{ug} \cdot \mathrm{kg}^{-1}$. Se notó una disminución en el porcentaje de inmovilidad entre ambos días de 
exposición $(t=2,93 ; \mathrm{p}=0,02)$. En relación al oscurecimiento se determinó que el LOEC fue de 49,5 ug. $\mathrm{kg}^{-1}$. Ambos días de exposición no presentaron diferencias en el patrón de efecto $(t=2,20 ; p=0,07)$. Los valores de $\mathrm{CE}_{50}$ a los 7 y 14 días para el parámetro adelgazamiento decrecieron para las concentraciones ensayadas. Se observaron diferencias estadísticamente significativas en los bioensayos segúnel tiempo de exposición $(\mathrm{t}=2,59 ; \mathrm{p}=0,04)$.

Para el parámetro estrangulación se observó un incremento en el porcentaje del parámetro estrangulación con el tiempo de exposición $(\mathrm{t}=3,36 ; \mathrm{p}=0,01)$. No se observaron valores de $\mathrm{CE}_{50}$ en los bioensayos sobre $E$. fetida en relación al fraccionamiento. Se determinó que el NOEC fue la concentración más alta utilizada durante el bioensayo. No se apreció un incremento en el porcentaje de fraccionamiento $(t=1,44 ; \mathrm{p}=0,20)$. El promedio de peso corporal empleado en el bioensayo con gasolina de 90 octanosPetroperú no varió en relación a las concentraciones evaluadas. Se observó un incremento en el porcentaje de peso húmedo con el tiempo de exposición $(\mathrm{t}=7,96 ; \mathrm{p}=0,00)$. Los resultados muestran que la lombriz de tierra $E$. fetida presenta una alta sensibilidad en relación a los parámetros de movilidad, oscurecimiento y adelgazamiento. Por otro lado, no se observaron efectos sobre la lombriz de tierra en los parámetros de mortalidad, estrangulación, fraccionamiento y peso corporal.

La ERA de la gasolina de 90 octanosPetroperú en base al NOEC, mostró que todos parámetros evaluados fueron sensibles y por lo tanto ayudaron a determinaron el riesgo, pues presentaron valores mayores a 1 (Tabla 4). La ERA de la gasolina de 90 octanos-Petroperú evaluada en base a la toxicidad de la concentración efectiva $\left(\mathrm{CE}_{50}\right)$, mostró que tres de los parámetros evaluados a los 7 y 14 días presentaron un riesgo para la lombriz de tierra E. fetida. Así, la secuencia relativa de mayor a menor en relación a los cocientes de riesgo de la gasolina de 90 octanos-Petroperú fue igual para ambos días de exposición: oscurecimiento > inmovilidad $>$ adelgazamiento. 
Tabla 3

Efectos letales y subletales de la gasolina de 90 octanos-Petroperú en seis parámetros porcentuales y en el peso corporal (mg) evaluados en Eisenia fetida.

\begin{tabular}{|c|c|c|c|c|c|c|c|c|c|c|c|c|c|c|c|}
\hline & $\begin{array}{r}\text { Mortal } \\
\%\end{array}$ & & $\begin{array}{r}\text { Inmol } \\
?\end{array}$ & vilidad & Oscure & imiento & $\begin{array}{l}\text { Adelgaz } \\
\%\end{array}$ & amiento & Estrang & & Fraccion & & & $\begin{array}{l}\text { so Corpo } \\
\text { (mg) }\end{array}$ & \\
\hline $\begin{array}{l}\text { Concentración } \\
\text { (ug A A*Kg-1) }\end{array}$ & $7 d$ & $14 d$ & $7 d$ & $14 d$ & $7 d$ & $14 d$ & $7 d$ & $14 d$ & $7 d$ & $14 d$ & $7 d$ & $14 d$ & $\begin{array}{c}\text { Peso } \\
\text { Húmedo } \\
7 d\end{array}$ & $\begin{array}{c}\text { Peso } \\
\text { Húmedo } \\
14 d \\
\end{array}$ & $\begin{array}{l}\text { Peso } \\
\text { Seco } \\
14 d \\
\end{array}$ \\
\hline Control Agua & $\mathrm{Oa}$ & $\mathrm{Oa}$ & $\mathrm{Oa}$ & $\mathrm{Oa}$ & $\mathrm{Oa}$ & $\mathrm{Oa}$ & $\mathrm{Oa}$ & $\mathrm{Oa}$ & $\mathrm{Oa}$ & 0 & $\mathrm{Oa}$ & 0 & $168,75 a$ & $158,46 a$ & $28,21 a$ \\
\hline $\begin{array}{c}\text { Control Agua t } \\
\text { Alcohol }\end{array}$ & $\mathrm{Oa}$ & $\mathrm{Oa}$ & $\mathrm{Oa}$ & $\mathrm{Oa}$ & $\mathrm{Oa}$ & $\mathrm{Oa}$ & $\mathrm{Oa}$ & $\mathrm{Oa}$ & $\mathrm{Oa}$ & 0 & $\mathrm{Oa}$ & 0 & $185,5 a$ & $161,25 a$ & $27 a$ \\
\hline 49,5 & $2,5 a b$ & $2,56 a$ & 22,860 & $8,57 a b$ & 34,296 & $37,14 b$ & $25,71 \mathrm{~b}$ & $7,14 a$ & $2,5 a$ & 0 & $\mathrm{Oa}$ & 0 & $172,31 \mathrm{a}$ & $143,16 \mathrm{a}$ & 29,21a \\
\hline 148,5 & $2,5 a b$ & $5,13 a$ & $42,86 \mathrm{bc}$ & $28,57 \mathrm{bc}$ & $48,57 \mathrm{bcd}$ & $48,57 b c$ & $37,14 b c$ & $21,43 a$ & $5 a$ & 0 & $\mathrm{Oa}$ & 0 & $182,82 a$ & $162,16 a$ & $27,84 a$ \\
\hline 346,5 & $10 b$ & $12,82 a$ & $57,14 c d$ & 42,86cd & $37,14 b c$ & $51,43 b c$ & $28,57 b$ & $28,57 a b$ & $5 a$ & 0 & $2,5 a$ & 0 & $165,28 \mathrm{a}$ & $151,76 a$ & $27,06 a$ \\
\hline 841,5 & $5 a b$ & $5,13 \mathrm{a}$ & $60 \mathrm{~cd}$ & $45,71 \mathrm{~cd}$ & $57,14 \mathrm{~cd}$ & 60cd & $57,14 c d$ & $53,57 b$ & $2,5 a$ & 0 & $\mathrm{Oa}$ & 0 & $177,37 \mathrm{a}$ & 156,22a & $27,57 a$ \\
\hline 2079 & $5 a b$ & $7,69 \mathrm{a}$ & $74,29 \mathrm{~d}$ & $57,14 d$ & $68,57 d$ & $74,29 d$ & $62,86 \mathrm{~d}$ & $57,14 b$ & $5 a$ & 0 & $5 a$ & 0 & $184,47 a$ & $156,94 a$ & $27,22 \mathrm{a}$ \\
\hline NOEC (ug IA Kg-1) & 2079 & 2079 & $<49,5$ & $<49,5$ & $<49,5$ & $<49,5$ & $<49,5$ & $<49,5$ & 2079 & & 2079 & - & 2079 & 2079 & 2079 \\
\hline LOEC (ug A $\cdot K g-1)$ & $>2079$ & $>2079$ & 49,5 & 49,5 & 49,5 & 49,5 & 49,5 & 49,5 & $>2079$ & . & $>2079$ & . & $>2079$ & $>2079$ & $>2079$ \\
\hline Levene & 12,83 & 1,84 & 2,81 & 0,94 & 0,84 & 1,17 & 2,29 & 0,04 & 5,61 & . & 19,71 & . & 0,97 & 1,61 & 0,41 \\
\hline Sig & 0 & 0,14 & 0,036 & 0,49 & 0,55 & 0,36 & 0,074 & 0,69 & 0,001 & . & 0 & . & 0,45 & 0,14 & 0,87 \\
\hline$F$ & 2,93 & 2 & 48,294 & 19,96 & 36,81 & 44,46 & 27,03 & 14,08 & 0,65 & . & 1,75 & . & 2,16 & 1,75 & 0,44 \\
\hline Sig & 0,031 & 0,11 & 0 & 0 & 0 & 0 & 0 & 0 & 0,69 & . & 0,16 & . & 0,047 & 0,11 & 0,85 \\
\hline CE(LI50 (ug A*Kg-1) & ND & ND & 299,9 & 974,23 & 277,84 & 213,99 & 603,11 & 1110 & $\mathrm{ND}$ & ND & ND & ND & ND & ND & ND \\
\hline
\end{tabular}

ND. Valor No determinado. d=día. En una misma fila, valores con letras minúsculas diferentes (a, b, c, d) son significativamente diferentes en la variación de los parámetros evaluados en los tratamientos (prueba de Tukey $\mathrm{p}<0,05$ ). IA: Ingrediente Activo (Xileno 6,6\%). Los porcentajes de los parámetros son los valores promedios para las cuatro réplicas. 
Tabla 4

Evaluación del riesgo ambiental terrestre de la gasolina ecológica de 90 octanos-Petroperú en base al xileno sobre Eisenia fetida.

\begin{tabular}{|c|c|c|c|c|c|c|c|c|c|}
\hline Especie & $\begin{array}{l}\text { Efecto } \\
\text { Tóxico }\end{array}$ & Parámetros & $\begin{array}{c}\text { Toxicidad } \\
\text { NOEC }\end{array}$ & Factor & $\begin{array}{l}\text { PNEC } \\
\text { NOEC }\end{array}$ & $\begin{array}{c}\text { Exposición } \\
\text { (ECA suelo) } \\
\left(\text { ug॰ } \mathrm{Kg}^{-1}\right)\end{array}$ & CR & LOC & $\begin{array}{l}\text { RIESGO } \\
\text { NOEC }\end{array}$ \\
\hline \multirow{9}{*}{$\begin{array}{c}\text { Eisenia } \\
\text { fetida }\end{array}$} & \multirow[t]{5}{*}{ Letal } & Mortalidad & 2079 & 100 & 20,79 & 11000 & 529,10 & 1.00 & SI \\
\hline & & Inmovilidad & 49,5 & 100 & 0,50 & 11000 & 22222,22 & 1.00 & SI \\
\hline & & Oscurecimiento & 49,5 & 100 & 0,50 & 11000 & 22222,22 & 1.00 & SI \\
\hline & & Adelgazamiento & 49,5 & 100 & 0,50 & 11000 & 22222,22 & 1.00 & $\mathrm{SI}$ \\
\hline & & Estrangulación & 2079 & 100 & 20,79 & 11000 & 529,10 & 1.00 & $\mathrm{Sl}$ \\
\hline & \multirow[t]{4}{*}{ Subletal } & Fraccionamiento & 2079 & 100 & 20,79 & 11000 & 529,10 & 1.00 & $\mathrm{SI}$ \\
\hline & & Peso humedo $7^{\circ}$ día & 2079 & 100 & 20,79 & 11000 & 529,10 & 1.00 & SI \\
\hline & & Peso húmedo $14^{\circ}$ día & 2079 & 100 & 20,79 & 11000 & 529,10 & 1.00 & $\mathrm{Sl}$ \\
\hline & & Peso seco & 2079 & 100 & 20,79 & 11000 & 529,10 & 1.00 & SI \\
\hline Especie & $\begin{array}{l}\text { Efecto } \\
\text { Tóxico }\end{array}$ & Parámetros & $\begin{array}{c}\text { Toxicidad } \\
\text { CE(L)50 }\end{array}$ & Factor & PNEC & $\begin{array}{c}\text { Exposición } \\
\text { (ECA suelo) } \\
\text { (ug・Kg-1) }\end{array}$ & CR & LOC & $\begin{array}{l}\text { RIESGO } \\
\text { NOEC }\end{array}$ \\
\hline \multirow{6}{*}{$\begin{array}{c}\text { Eisenia } \\
\text { fetida }\end{array}$} & \multirow{6}{*}{ Subletal } & Inmovilidad $7^{\circ}$ día & 299,9 & 1000 & 0,30 & 11000 & 36678,89 & 1.00 & $\mathrm{SI}$ \\
\hline & & Oscurecimiento $7^{\circ}$ día & 277,84 & 1000 & 0,28 & 11000 & 39591.13 & 1.00 & SI \\
\hline & & Adelgazamiento $7^{\circ}$ día & 603,11 & 1000 & 0,60 & 11000 & 18238,80 & 1.00 & SI \\
\hline & & Inmovilidad 14 día & 974,23 & 1000 & 0,97 & 11000 & 11290,97 & 1.00 & $\mathrm{SI}$ \\
\hline & & Oscurecimiento $14^{\circ}$ día & 213,99 & 1000 & 0,21 & 11000 & 51404,27 & 1.00 & $\mathrm{SI}$ \\
\hline & & Adelgazamiento $14^{\circ}$ día & 1110 & 1000 & 1,11 & 11000 & 9909,91 & 1.00 & $\mathrm{SI}$ \\
\hline
\end{tabular}

Toxicidad=NOEC=Concentración másaltaen la queno se observa efecto. Exposición=ECAsuelo=Estándar de Calidad Ambiental de Suelo DS Nº02-2013-MINAM-Perú para el xileno. Factor de seguridad=100 para NOEC y 1000 para $\mathrm{CE}_{50}$. PNEC= Concentración pronosticada sin efecto= NOEC/Factor de seguridad. $\mathrm{CR}=$ Cociente de Riesgo= Exposición/PNEC. LOC= Nivel crítico.

\section{Gasolina de 90 Octanos - Repsol, expresada en base al xileno (ug. $\mathrm{kg}^{-1}$ )}

La Tabla 5 muestra los efectos de la mortalidad, inmovilidad, oscurecimiento, adelgazamiento, estrangulación, fraccionamiento y peso sobre E. fetida por efecto de gasolina de 90 octanos-Repsol y expresadas en ug de xileno.IA $\cdot \mathrm{kg}^{-1}$. No se observó variciones en la mortalidad a los 7 y 14 días de exposición $(t=2,20$; $\mathrm{p}=0,07)$. Se determinó que el NOEC fue la concentración más alta utilizada durante el bioensayo. Para la inmovilidad se registraron diferencias en los valores de $\mathrm{CE}_{50}$ entre los 7 y 14 días $(\mathrm{t}=1,95$; $\mathrm{p}=0,09)$. No se notó diferencias en el oscurecimiento según el tiempo de exposición $(t=1,55 ; \mathrm{p}=0,17)$. Los valores de $\mathrm{CE}_{50}$ a los 7 y 14 días para el parámetro adelgazamiento decrecieron para las concentraciones ensayadas. Para ambos días de exposición presentaron un patrón de efecto diferente $(t=6,82 ; \mathrm{p}<0,05)$.

Para el parámetro estrangulación, no se observó cambio con el tiempo de exposición $(t=2,29 ; \mathrm{p}=0,06)$. No se vieron diferencias para la $\mathrm{CE}_{50}$ de E. fetida en relación al fraccionamiento entre los $7 \mathrm{y}$ 
14 días de exposición $(t=1,33 ; \mathrm{p}=0,23)$. El peso corporal empleado en el bioensayo con gasolina de 90 octanos-Repsol varió en relación a las concentraciones. Se observó un aumento en el peso húmedo con el tiempo de exposición $(t=17,14 ; p=0,00)$. Se vio sensibilidad en relación a movilidad, oscurecimiento y adelgazamiento.

Al comparar entre ambas gasolinas de 90 octanos ecológicas, se obtuvo que la mayor toxicidad de la gasolina-
Petroperú en la inmovilidad $\left(\mathrm{CE}_{50}=\right.$ $\left.974,23 \mathrm{ug} \cdot \mathrm{kg}^{-1}\right)$ y en la gasolina-Repsol en el adelgazamiento $\left(\mathrm{CE}_{50}=418,41\right.$ ug. $\left.\mathrm{kg}^{-1}\right)$. La gasolina de Repsol en general es más tóxica que la de Petroperú. La ERA en base al NOEC, mostró que los parámetros evaluados determinaron riesgo al presentar valores superiores al LOC equivalente a 1 . Hay riesgo para la gasolina de 90 octanos-Repsol en la inmovilidad, oscurecimiento, adelgazamiento y peso húmedo de $E$. fetida. (Tabla 6).

\section{Tabla 5}

Efectos letales y subletales de la gasolina de 90 octanos-Repsol en seis parámetros porcentuales y en el peso corporal (mg) evaluados en Eisenia fetida.

\begin{tabular}{|c|c|c|c|c|c|c|c|c|c|c|c|c|c|c|c|}
\hline & Morta & & & & $\begin{array}{r}\text { Oscureci } \\
\%\end{array}$ & cimiento & $\begin{array}{r}\text { Adelgaza } \\
\%\end{array}$ & zamiento & $\begin{array}{r}\text { Estrang } \\
?\end{array}$ & ylación & $\begin{array}{r}\text { Fraccion } \\
\%\end{array}$ & & & $\begin{array}{c}\text { eso Corpo } \\
(\mathrm{mg})\end{array}$ & \\
\hline $\begin{array}{l}\text { Concentración } \\
\text { (ug Arkg-1) }\end{array}$ & $7 d$ & $14 \mathrm{~d}$ & $7 d$ & $14 \mathrm{~d}$ & $7 d$ & $14 \mathrm{~d}$ & $7 d$ & $14 \mathrm{~d}$ & $7 d$ & $14 d$ & $7 d$ & $14 d$ & $\begin{array}{l}\text { Peso } \\
\text { Húmedo } \\
7 d\end{array}$ & $\begin{array}{c}\text { Peso } \\
\text { Húmedo } \\
140\end{array}$ & $\begin{array}{l}\text { Peso } \\
\text { Seco } \\
14 d\end{array}$ \\
\hline Control Agua & $\mathrm{Oa}$ & $\mathrm{Oa}$ & $\mathrm{Oa}$ & $\mathrm{Oa}$ & $\mathrm{Oa}$ & $\mathrm{Oa}$ & $\mathrm{Oa}$ & $\mathrm{Oa}$ & $\mathrm{Oa}$ & $\mathrm{Oa}$ & $\mathrm{Oa}$ & 0 & $203,50 \mathrm{a}$ & $157,75 a$ & $25,5 \mathrm{a}$ \\
\hline $\begin{array}{c}\text { Control Agua + } \\
\text { Acohol }\end{array}$ & $\mathrm{Oa}$ & $\mathrm{Oa}$ & $\mathrm{Oa}$ & $\mathrm{Oa}$ & $\mathrm{Oa}$ & $\mathrm{Oa}$ & $\mathrm{Oa}$ & $\mathrm{Oa}$ & $\mathrm{Oa}$ & $\mathrm{Oa}$ & $\mathrm{Oa}$ & 0 & $215,75 a$ & 174,25ab & $25,5 a$ \\
\hline 49.5 & $2,5 \mathrm{a}$ & $2,5 a$ & 42,56 & 27,030 & $37,14 b$ & $36,36 b$ & 26,476 & $56,77 b$ & $\mathrm{Oa}$ & $\mathrm{Oa}$ & $\mathrm{Oa}$ & 0 & $218,97 a$ & $184,87 b$ & $23,33 \mathrm{a}$ \\
\hline 148.5 & $2,5 \mathrm{a}$ & $7,5 \mathrm{a}$ & $52,50 \mathrm{c}$ & $32,430 \mathrm{c}$ & $51,436 c$ & $45,45 b c$ & $44,12 b c$ & 63,330 & $5 a$ & $\mathrm{Oa}$ & $\mathrm{Oa}$ & 0 & $219,74 a$ & $183,78 a b$ & $25,68 \mathrm{a}$ \\
\hline 346.5 & $7,5 \mathrm{a}$ & 10a & 60cd & $45,950 \mathrm{c}$ & c 51,430c & $48,480 \mathrm{c}$ & $50 \mathrm{dd}$ & 63,336 & $2,5 a$ & $\mathrm{Oa}$ & $\mathrm{Oa}$ & 0 & $225,68 \mathrm{a}$ & $174,72 a b$ & $23,89 \mathrm{a}$ \\
\hline 841.5 & $\mathrm{Oa}$ & $10 \mathrm{a}$ & $62,5 \mathrm{~cd}$ & 48,650 & $600 \mathrm{c}$ & $60,61 c$ & $64,71 d$ & $80 b$ & $10 \mathrm{a}$ & $\mathrm{Oa}$ & $2,5 a$ & 0 & $218,75 a$ & 184,176 & $23,33 \mathrm{a}$ \\
\hline 2079 & $\mathrm{Oa}$ & $12,5 \mathrm{a}$ & $70 \mathrm{~d}$ & $51,35 \mathrm{c}$ & 65,710 & $63,64 \mathrm{c}$ & $52,94 \mathrm{~cd}$ & 83,336 & $12,5 a$ & $2,5 a$ & $7,5 \mathrm{a}$ & 0 & $227,25 \mathrm{a}$ & 185,140 & $23,94 a$ \\
\hline $\operatorname{NOEC}(\operatorname{ug} A \cdot K g-1)$ & 2079 & 2079 & $<49,5$ & $<49,5$ & $<49,5$ & $<49,5$ & $<49,5$ & $<49,5$ & 2079 & 2079 & 2079 & . & 2079 & 346,5 & 2079 \\
\hline LOEC (ug $A * K g-1)$ & $>2079$ & $>2079$ & 49,5 & 49,5 & 49,5 & 49,5 & 49,5 & 49,5 & $>2079$ & $>2079$ & $>2079$ & . & $>2079$ & 2841,5 & $>2079$ \\
\hline Levene & 8,36 & 8,77 & 2,57 & 0,73 & 1,72 & 0,77 & 2,5 & 1,67 & 31,73 & 9 & 10,64 & . & 3,79 & 1,42 & 0,6 \\
\hline Sig & 0 & 0 & 0,05 & 0,63 & 0,17 & 0,6 & 0,06 & 0,18 & 0 & 0 & 0 & . & 0,001 & 0,21 & 0,73 \\
\hline$F$ & 1,53 & 1,39 & 70,49 & 24,23 & 28,38 & 32,5 & 47,5 & 25,69 & 1,78 & 1 & 1,93 & - & 1,65 & 2,69 & 0,63 \\
\hline Sig & 0,22 & 0,27 & 0 & 0 & 0 & 0 & 0 & 0 & 0,15 & 0,45 & 0,12 & . & 0,13 & 0,015 & 0,7 \\
\hline$E(L / 50(\operatorname{lgg} A \cdot K g-1)$ & ND & $\mathrm{ND}$ & 117,59 & 1194 & 207,04 & 300,21 & 418,41 & 43,67 & ND & $N D$ & ND & ND & ND & ND & ND \\
\hline
\end{tabular}

ND. Valor No determinado. d=día. En una misma fila, valores con letras minúsculas diferentes (a, b, c, d) son significativamente diferentes en la variación de los parámetros evaluados en los tratamientos (prueba de tukey p $<0,05$ ). IA: Ingrediente Activo (Xileno 6,6\%). Los porcentajes de los parámetros son los valores promedios para las cuatro réplicas. 
Tabla 6

Evaluación del riesgo ambiental terrestre de la gasolina ecológica de 90 octanos-Repsol en base al xileno sobre Eisenia fetida.

\begin{tabular}{|c|c|c|c|c|c|c|c|c|c|}
\hline Especie & $\begin{array}{l}\text { Efecto } \\
\text { Tóxico }\end{array}$ & Parámetros & $\begin{array}{c}\text { Toxicidad } \\
\text { NOEC }\end{array}$ & Factor & $\begin{array}{l}\text { PNEC } \\
\text { NOEC }\end{array}$ & $\begin{array}{c}\text { Exposición } \\
\text { (ECA suelo) } \\
\left(\mathrm{ug}^{\circ} \mathrm{Kg}^{-1}\right)\end{array}$ & CR & LOC & $\begin{array}{c}\text { RIESGO } \\
\text { NOEC }\end{array}$ \\
\hline \multirow{9}{*}{$\begin{array}{l}\text { Eisenia } \\
\text { fetida }\end{array}$} & Letal & Mortalidad & 2079 & 100 & 20,79 & 11000 & 529,10 & 1.00 & SI \\
\hline & \multirow{8}{*}{ Subletal } & Inmovilidad & 49,5 & 100 & 0,50 & 11000 & 22222,22 & 1.00 & SI \\
\hline & & Oscurecimiento & 49,5 & 100 & 0,50 & 11000 & 22222,22 & 1.00 & SI \\
\hline & & Adelgazamiento & 49,5 & 100 & 0,50 & 11000 & 22222,22 & 1.00 & SI \\
\hline & & Estrangulación & 2079 & 100 & 20,79 & 11000 & 529,10 & 1.00 & SI \\
\hline & & Fraccionamiento & 2079 & 100 & 20,79 & 11000 & 529,10 & 1.00 & $\mathrm{SI}$ \\
\hline & & Peso húmedo 7 días & 2079 & 100 & 20,79 & 11000 & 529,10 & 1.00 & SI \\
\hline & & Peso húmedo 14 días & 346,5 & 100 & 3,47 & 11000 & 3174,60 & 1.00 & SI \\
\hline & & Peso seco & 2079 & 100 & 20,79 & 11000 & 529,10 & 1.00 & SI \\
\hline Especie & $\begin{array}{l}\text { Efecto } \\
\text { Tóxico }\end{array}$ & Parámetros & $\begin{array}{c}\text { Toxicidad } \\
\text { CE(L)50 }\end{array}$ & Factor & PNEC & $\begin{array}{c}\text { Exposición } \\
\text { (ECA suelo) } \\
(\text { ug・Kg-1) }\end{array}$ & CR & LOC & $\begin{array}{l}\text { RIESGO } \\
\text { NOEC }\end{array}$ \\
\hline \multirow{6}{*}{$\begin{array}{c}\text { Eisenia } \\
\text { fetida }\end{array}$} & \multirow{6}{*}{ Subletal } & Inmovilidad $7^{\circ}$ día & 117,59 & 1000 & 0,11759 & 11000 & 93545,37 & 1.00 & SI \\
\hline & & Oscurecimiento $7^{\circ}$ día & 207,04 & 1000 & 0,20704 & 11000 & 53129,83 & 1.00 & SI \\
\hline & & Adelgazamiento $7^{\circ}$ día & 418,41 & 1000 & 0,41841 & 11000 & 26290,00 & 1.00 & $\mathrm{SI}$ \\
\hline & & Inmovilidad $14^{\circ}$ día & 1194 & 1000 & 1194.00 & 11000 & 9212,73 & 1.00 & $\mathrm{SI}$ \\
\hline & & Oscurecimiento $14^{\circ}$ día & 300,21 & 1000 & 0,30021 & 11000 & 36641,02 & 1.00 & $\mathrm{SI}$ \\
\hline & & Adelgazamiento $14^{\circ}$ día & 43,67 & 1000 & 0,04367 & 11000 & 251889,17 & 1.00 & SI \\
\hline
\end{tabular}

Toxicidad $=$ NOEC $=$ Concentración másalta en laqueno seobservaefecto. Exposición=ECAsuelo=Estándar de Calidad Ambiental de Suelo DS Nº02-2013-MINAM-Perú para el xileno. Factor de seguridad= 100 o 1000 (Este valor es inversamente proporcional a la cantidad y calidad de los datos de toxicidad); (100) para NOEC y (1000) para $\mathrm{CE}_{50}$. PNEC=Concentración pronosticada sin efecto=NOEC/Factor de seguridad. $\mathrm{CR}=$ Cociente de Riesgo= Exposición/PNEC. LOC= Nivel crítico comparativo.

\section{Discusión}

De los bioensayos realizados con la gasolina en base al xileno, no se observaron efectos letales significativos en la mortalidad de E. fétida. Nuestros resultados son similares a Bamgbose \& Anderson (2020) encontraron que los biodiesel y diesel en E. fetida fue menos tóxicos en ensayos de mortalidad que en las respuestas subletales. da Silva Júnior et al. (2018) no observaron cambios en la mortalidad en las lombrices de tierra por acción del BTEX (benceno, tolueno, etilbenceno y xileno). Abdollahinejad et al. (2020) observaron tasas de sobrevivencia razonables de la lombriz de tierra por acción de suelo contaminados por derivados de petróleo. En contraste no concuerda con lo presentado por Gómez (2014), quien evaluó la mortalidad de este invertebrado en suelos contaminados por pozos petroleros y de refinerías de petróleo, encontrando efecto en la toxicidad por letalidad.

Otros autores como Kobeticová et al. (2008) registraron mortalidad por los HAP (Hidrocarburos Aromáticos Policíclicos) en E. fetida después de cuatro semanas de 
exposición. Pasková et al. (1988) y Droge et al. (2006) observaron una alta toxicidad de los HAP en la biota del suelo. Uno de las razones por los cuales no se registraron efectos letales en $E$. fetida podría deberse a las características de los BTEX presentes en la gasolina, debido a que la misma al contacto con el suelo superficial actúa por volatilización, disolución o la adsorción de componentes individuales según sus propiedades físicas/químicas (ATSDR, 1995).

La identificación de los efectos de la toxicidad subletal es de suma importancia, debido a que nos ayuda a determinar el estado de la lombriz de tierra, pues estos se ven reflejados en su crecimiento y reproducción a mediano plazo (Radamass et al., 2015; da Silva Júnior et al., 2018; Bamgbose \& Anderson, 2020). Se observaron efectos subletales para la inmovilidad sobre E. fetida. Este se podría explicar por una reducción en la producción de la mucosidad que disminuye el desplazamiento de la lombriz (Weeks \& Comber, 2005). Tang et al. (2016) encontraron que los tóxicos en el suelo ocasionan una reducción del enterramiento e inhibición en el ritmo respiratorio.

De los tres parámetros más sensibles, el oscurecimiento a los 14 días de exposición resultó ser un sensible a la gasolina-Petroperú. An \& Lee (2008) registraron que la exposición corporal es más tóxica que la exposición a tóxicos por ingesta en la lombriz de tierra. Roubalová et al. (2015) aseveraron que cuando se acumulan los contaminantes se pueden romper células y tejidos, por la reducción progresiva de las vellosidades intestinales y del tejido cloragógeno en las lombrices de tierra. Salanitro et al. (1997) indican que las respuestas bioquímicas como el estrés oxidativo y la genotoxicidad a los tóxicos en las lombrices de tierra todavía no han sido investigadas a profundidad.

El adelgazamiento en $E$. fetida está ligado a la pérdida de fluidos celómicos tal como lo afirma Radamass et al. (2015) quienes compararon dos tipos de aceites de motor (fresco y usado) y encontraron toxicidad en base a la inactividad, fragmentación, hinchazón y expulsión de fluido celómico antes de la muerte. Curry (1994), indica que los contaminantes más pesados conducen a efectos negativos en la lombriz, produciendo problemas como la pérdida de peso (adelgazamiento) debido a que prefieren morir de inanición en lugar de alimentarse de suelos contaminados (Radamass et al., 2015). Bamgbose \& Anderson (2020) observaron en E. fetida respuestas de morbilidad como descarga excesiva de fluídos celómicos por acción de compuestos biodiesel y diesel.

Para los tres parámetros más sensibles también se observó que el valor de la concentración más baja de NOEC $\left(<49,5 \mathrm{ug} \cdot \mathrm{kg}^{-1}\right)$ generó los efectos más representativos tanto para la gasolina de 90 octanos-Petroperú como para la gasolina de 90 octanos-Repsol. Los parámetros subletales reflejan efectos que no se pueden observar con las pruebas de toxicidad letal (Dominique et al., 2007; Kuppusamy et al., 2020). Dawson et al. (2007) sugieren la incorporación de pruebas subletales para las lombrices de tierra proporcionan datos sensibles y ecológicamente relevantes (Lowe \& Butt, 2007).

Los parámetros de estrangulación $\mathrm{y}$ fraccionamiento no influyeron de 
manera significativa en la lombriz de tierra. Venkateswara et al. (2003), afirman que la segmentación ocurre por el agotamiento de fluido celómico. Eom et al. (2007) muestran por efectos subletales sensibilidad de E. fetida a suelos contaminados con hidrocarburos aromáticos. Venkateswara et al. (2003) adicionan que la exposición al tetraetilo de plomo (TEL) y óxido de plomo (un aditivo de la gasolina) provoca rupturas de la cutícula y la piel, extrusión de fluido celómico y segmentación. Bamgbose \& Anderson (2020) encontraron en E. fetida respuestas de fragmentación anterior y posterior por exposición a compuestos biodiesel y diésel.

El peso húmedo y el peso seco no ocasionaron toxicidad en el oligoqueto. Bamgbose \&Anderson (2020) observaron en la lombriz de tierra disminución del peso por efecto del biodiesel y diésel. Liu et al. (2010) afirman que los contaminantes hidrofílicos pueden entran en el cuerpo de lombriz a través de la piel predominantemente, mientras que las sustancias hidrofóbicas entran a través del tracto digestivo. Lo resultados evidencian que las gasolinas ecológicas no afectan la ingestión y la asimilación de las lombrices lo cual se vio evidenciado en la ausencia de efecto en el peso de las lombrices. Radamass et al. (2015) afirman que las enzimas deshidrogenasas y nitrificadoras de los microorganismos de suelo tienden a controlar los efectos de los HTP sobre la toxicidad del suelo. Spurgeon et al. (2003) y Labud et al. (2006), corroboran que la toxicidad de los hidrocarburos depende del tipo y concentración de esta sustancia química, capacidad de intercambio catiónico, $\mathrm{pH}$ y contenido de materia orgánica del suelo (Wang et al., 2000; Song et al., 2005; Hund-Rinke \& Wiechering, 2011; Palafox et al., 2012; Whitfield et al., 2013; Kuppusamy et al., 2020), lo que puede ocasionar cambios respuestas bioquímica a nivel de las enzimas antioxidantes POD (peroxidasa), CAT (catalasa) y SOD (superóxido dismutasa) (Liu et al., 2010) y en la actividad de las células similares a natural killer $(\mathrm{NK})$ que forman parte del sistema de defensa en $E$. fetida (Patel et al., 2007).

Las concentraciones evaluadas, se determinaron considerando al xileno como el ingrediente activo. Si bien el benceno es la base estructural de los hidrocarburos aromáticos totales (HAT), el xileno causa toxicidad en suelos, debido a que es el más abundante y el más representativo en la composición de la gasolina. Pérez et al. (2015) afirman que el xileno presenta agregados y el componente "menos volátil" es el o-xileno, debido a que no hay pérdida de concentración en el tiempo, y por lo tanto es un contaminante que afecta al suelo. Desafortunadamente, la concentración exacta de los BTEX en gasolina que se liberan al medio ambiente por lo general no se conoce con exactitud (PES, 1999). Cline et al. (1991) observaron que la composición específica de la gasolina variará dependiendo del origen del petróleo, el método de producción, de la ubicación de uso final y de la estación del año.

La gasolina de 90 octanos-Petroperú utiliza un crudo proveniente de cañolimón (Colombia) (Petroperú, 2013), y la gasolina de 90 octanos-Repsol es elaborada en base a un crudo africano, que son considerados altamente cargados en azufre (Repsol, 2007). Podemos indicar que sus componentes químicos podrían ser diferentes y por lo tanto, 
ocasionar valores de toxicidad subletal diferentes en la lombriz de tierra (Lemus et al., 2020). El xileno es el compuesto de mayor persistencia en suelo (ASTDR, 1995; Pinedo, 2014). Pero al mismo tiempo no podemos adjudicar todos los efectos tóxicos únicamente al xileno, porque los cuatro componentes del BTEX pueden ocasionar impacto por efecto de mezcla generando un efecto sinérgico o antagónico (Smith, 2010). Tas et al. (1997) durante un ensayo de 14 días determinaron que las lombrices de tierra al contacto con el xileno presente en jabones y detergentes no generaron efectos adversos en la supervivencia y comportamiento de las lombrices.

La disminución de la concentración letal durante los bioensayos podría verse disminuida por la adición de agua, debido a que para mantener la humedad óptima durante los bioensayos se adicionó 75 $\mathrm{mL}$ de agua continuamente. Donaldson et al. (1992) indican que la volatilidad de la gasolina de suelos contaminados puede mejorar mediante la adición de agua al suelo, aunque esto va a depender de la porosidad es el suelo (Bossert $\&$ Bartha, 1984). Se ha aseverado que los compuestos aromáticos contienen grupos funcionales que son resistentes a la hidrólisis (Lyman et al., 1990; Khan et al., 2018). La capacidad de los compuestos BTEX para disolverse en agua afectará su biodisponibilidad en medio ambiente (Luo et al., 1999).

Se ha indicado que la gasolina mezclada con etanol genera una menor contaminación por derrame en suelo debido a que es mucho más volátil. Sin embargo, los datos obtenidos en al presente estudio nos muestran lo contrario, aun realizando la dilución del contaminante (gasolina de 90 octanos) en alcohol etílico de $96^{\circ}$, se han observado parámetros sensibles en E. fetida (Repsol, 2007; AGESP, 2013). La refinería La Pampilla utiliza para la gasolina-Repsol al $7,8 \%$ de etanol en su composición, por lo tanto se podría asumir que si bien en el control de etanol los resultados fueron iguales que el control de agua durante los bioensayos, la mezcla de etanol y gasolina de 90 octanos podría ser otro factor que exacerbe los efectos subletales en la lombriz.

La principal fuente de contaminación por hidrocarburos (gasolinas) en el Perú son las estaciones de servicio clandestinas las mismas que no cuentan con planes de manejo y protección (planes de contingencia), y tienen a su cargo dispensadores viejos en las islas de despacho generando derrames, filtraciones y volcaduras de combustibles. Estas distribuidoras se encuentran ubicadas en zonas periféricas de Lima (OSINERGMIN, 2015). Es necesario tener una mayor supervisión y fiscalización debido a que el ingreso del crudo altera las propiedades del suelo y afecta su equilibrio biológico (Das $\&$ Chandran, 2011; Shukry et al., 2013).

\section{Conclusiones}

En la presente investigación no se observó efecto tóxico letal en las dos gasolinas ecológicas de 90 octanos evaluadas sobre la lombriz de tierra E. fetida en base al xileno. Los tres parámetros subletales más sensibles a la gasolina de 90 octanos-Petroperú y Repsol sobre la lombriz de tierra fueron la inmovilidad, el oscurecimiento, y el adelgazamiento. No se observaron efectos en la estrangulación, fraccionamiento y 
peso corporal. Según la ERA realizada para la gasolina ecológicas de 90 octanosPetroperú en base al factor de seguridad y el cociente de riesgo considerando el ECA-peruano para suelos equivalente a $11 \mathrm{mg} \cdot \mathrm{kg}^{-1}$, mostró que la inmovilidad, oscurecimiento y adelgazamiento fueron los más sensibles en la lombriz de tierra. Estos tres parámetros evaluados en relación al NOEC dieron un cociente de riesgo que superó el LOC equivalente a 1. Lo mismo se presentó en la evaluación de estos tres parámetros más sensibles teniendo en consideración la $\mathrm{CE}_{50}$. Por otro lado, la ERA realizada para la gasolina de 90 octanos-Repsol demostró que estos tres parámetros fueron sensibles al ser evaluados en función al NOEC, al igual que la gasolina de 90 octanos-Petroperú. Finalmente, el peso húmedo a los 14 días también presentó un riesgo significativo en la lombriz al igual que la inmovilidad, oscurecimiento y adelgazamiento evaluados en base a la $\mathrm{CE}_{50}$.

\section{Referencias}

Abdollahinejad, B., Pasalari, H., Jafari, A.J., Esrafili, A. \& Farzadki, M. (2020). Bioremediation of diesel and gasoline-contaminated soil by covermicomposting amended with activated sludge: Diesel and gasoline degradation and kinetics. Environmental Pollution, 263, 114584.

AGESP (Asociación de Grifos y Estaciones de Servicio del Perú) (2013). Asociación de Grifos y Estaciones de Servicio. Boletín Informativo, Consultado de http:// www.agesp.com

Alonzo, L. \& Chicas. S. (2013). Determinación de la concentración letal $50\left(\mathrm{CL}_{50}\right)$ de dos plaguicidas sintéticos utilizando Eisenia foetida (lombriz de tierra roja californiana) en el cultivo de Cucumis sativus $\mathrm{L}$ (pepino) en el distrito de riego del valle de zapotitan, La Libertad. El Salvador: Universidad del Salvador.

An, Y. \& Lee, W. (2008). Comparative and combined toxicities of toluene and methyl tert-butyl ether to an
Asian earthworm Perionyx excavatus. Chemosphere, 71, 407-411.

ATSDR (Agency for Toxic Substances and Disease Registry) (1995). Toxicological Profile for Gasoline. Agency for Toxic Substances and Disease Registry. US Department of Health and Human Services, pp. 1-224.

Bamgbose, I.A. Anderson, T.A. 2020. Ecotoxicity of three plant-based biodiesels and diesel using, Eisenia fetida. Environmental Pollution, 260, 113965.

Bautista-Medina, Y.B. \& Iannacone, J. 2020. Toxicidad del lodo de perforación minera en el bioindicador Porcellio laevis (Latreille, 1804) (Crustacea: Isopoda). Paideia XXI, 10, 95-119.

Bossert, I. \& Bartha , R. (1984). The fate of petroleum in soil ecosystems. Atlas, $R M$ c(Ed). Petroleum microbiology. Macmillan, New York. pp. 435-473.

Boyd, W.A., Stringer, V.A. \& Williams, P.L. (2001). Metal $\mathrm{LC}_{50}$ of a soil nematode 
compared to published Earthworm Data. Environmental Toxicology and Risk Assessment: science, policy and standardization- Implications for Environmental Decisions. Greenberg, B.M., Hull, R.N., Roberts, M.H. Jr. Gensemer, R.W. Eds., American Society for Testing and Materials, Standard Technical Publication 1403. 10: 2232352001 10, pp. 223-235.

Bravo, E. (2007). Los impactos de la explotación petrolera en ecosistemas tropicales y la biodiversidad. Ecuador: Acción Ecológica.

Brown, P.J., Long, S.M., Spurgeon, D.J., Svendsen, C. \& Hankard, P.K. (2004). Toxicological and biochemical responses of the earthworm Lumbricus rubellus to pyrene, a non-carcinogenic polycyclic aromatic hydrocarbon. Chemosphere, 57, 1675-1681.

Cline, P., Delfino, J. \& Rao, P. (1991). Partitioning of aromatic constituents into water from gasoline and other complex solvent mixtures. Environmental Science \& Technology, 25, 914-920

Cuevas, M., Ferrera, R., Roldán, A. \& Rodriguez, R. (2008a). Monitoreo de suelos contaminados mediante pruebas ecotoxicológicas. Metodos Ecotoxicologicos para la evaluación de suelos contaminados con hidrocarburos, pp. 211223. Consultado: https://www. eumed.net/rev/tlatemoani/11/ suelos-contaminados-pruebasecotoxicologicas.html

Curry, J. (1994). Grassland Invertebrates: Ecology, Influence on soil fertility, and effects on plant growth. Springer. New York.

Das, N. \& Chandran, P. (2011). Microbial degradation of petroleum hydrocarbon contaminants: an overview. Biotechnology Research International, 2011, Article 941810.

da Silva Júnior, F.M.R., Mendonça, F.S., Volcão, L.M. \& Honscha, L.C. (2018). Ecotoxicological assessment of BTEX to soil organisms using a terrestrial microcosm: multispecies soil system (MS-3). International Journal of Environmental Science and Technology, doi.org/10.1007/ s13762-018-2053-8

Delgado-Blas, V. \&Uc-Peraza, R.G. (2015). Acute toxicity and risk assessment of three commercial detergents using the polychaete Capitella sp. C from Chetumal Bay, Quintana Roo, Mexico. International Aquatic Research, 7, 251-261

Dominique, E., Ogusuku, E., Devine, G. \& Furlong, M. (2007). Uso de insecticidas: contexto y consecuencias ecológicas. Revista Peruana de Medicina experimental y Salud Pública, 25, 74-100

Donaldson, S., Miller, G. \& Miller, W. (1992). Remediation of gasoline-contaminated soil by passive volatilization. Journal of Environmental Quality, 21, 94-102.

Droge, S., Leon, P., Bleeker, E., Kraak, M. \& Gestel, C. (2006). Chronic toxicity of polycyclic aromatic compounds to the springtail Folsomia candida and Enchytraeus cryp- 
ticus. Environmental Toxycology and Chemistry, 25, 2423-2431.

EPA. (1998). Guidelines for Ecological Risk Assessment. United States: US Environmental Protection Agency. Washington, D.C. 188 p.

Escobar-Chávez, C., Alvariño, L. \& Iannacone, J. (2019). Evaluación del riesgo ambiental acuático de la mezcla de los plaguicidas imidacloprid (insecticida) y propineb (fungicida) en Daphnia magna Straus, 1820. Paideia XXI, 9, 301-322.

Flores, J. \& Bitteri, A. (2000). La Biotecnología en el impacto ambiental y su aplicación en el Perú. Ingeniería Industrial, 23, 105-119.

Flores, M.A., Torras, S., \& Téllez, R. (2004). Medidas de mitigación para uso de suelos contaminados por derrames de hidrocarburos en infraestructura de transporte terrestre. Publicación técnica, 257, 24-72.

Gomez, A. (2014). Evaluación de la Toxicología de suelos mediante un bioensayo con la lombriz de tierra Eisenia fetida. Bogotá, Colombia: Universidad Nacional de Colombia.

Hund-Rinke. K. \& Wiechering. H. (2011). Earthworm avoidance test for soil assessments. Journal of Soils and Sediments, 1, 15-20.

Iannacone, J. \& Alayo, M. (2002). Ensayos ecotoxicológicos con petroleo crudo, Diésel 2 y Diésel 6 con dos subespecies de Brachionus plicatilis Muller 1786 (Rotifera: Monogononta). Gayana, 66, 45-58.

Iannacone, J. \& Alvariño, L. (2004). Eisenia foetida (Savigny, 1826) como modelo ecotoxicologico para evaluar lindano y clorpirifos. Acta Zoológica Lilloana, 48, 5-12.

Iannacone, J. \& Alvariño, L. (2005). Selectividad del insecticida Cartap empleando bioensayos con organismos no destinatarios. Ecología Aplicada, 4, 1-14.

Iannacone, J. \& Alvariño, L. (2006). Eisenia foetida (Savigny, 1826) (Annelida:Lumbricidae) como modelo ecotoxicologico para evaluar lindano y clorpiños. Acta Zoológica Lilloana, 48, 5-12.

Iannacone, J., Alvariño, L. \& Paredes, C. (2009). Evaluación del riesgo ambiental del arseniato de plomo en bioensayos con ocho organismos no destinatarios. Journal of the Brazilian Society of Ecotoxicology, 4, 73-82.

IHCP. (2003). Technical Guidance Document on Risk Assessment. Ispra: Institute for Health and Consumer Protection. European Chemicals Bureau.

Kammenga, J., Dallinger, R., Donker, M., Kohler, H., Simonsen, V. \& Triebskorn, R. (2000). Biomarkers in terrestrial invertebrates for ecotoxicological soil risk assessment. Reviews of Environmental Contamination and Toxicology, 164, 93-147.

Khan, M.A.I., Biswas, B., Smith, E., Naidu, R. \& Megharaj, M. (2018). 
Toxicity assessment of fresh and weathered petroleum hydrocarbons in contaminated soil- a review. Chemosphere, 212, 755-767.

Kobeticová, K., Bezchlebová, J., Lána, J., Sochová, I. \& Hofman, J. (2008). Toxicity of four nitrogen-heterocyclic polyaromatic hydrocarbons (NPAHs) to soil organisms. Ecotoxicology and Environmental Safety, 71, 650-660.

Kuppusamy, S., Maddela, N.R., Megharaj, M. \& Venkateswarlu, K. (2020). Ecological Impacts of Total Petroleum Hydrocarbons. In: Total Petroleum Hydrocarbons. Springer, Cham.

Labud, V., Garcia, C. \& Hernandez, H. (2006). Effect of hydrocarbon pollution on the microbial properties of a sandy a clay soil. Chemosphere, 66, 1863-1871.

Lemos, G.S., Souza, M.S.S., Aleixo, N.V.S., Lima, E.N. \& Cotta, J.A.O. (2020). Ecotoxicological tests with Eisenia fetida for the evaluation of areas allegedly contqaminated with oil derivates in municipality of João Monlevade/MG. Research, Society and Development, 9, e115963025.

Liu, Y., Zhou, Q., Xie, X., Lin, D. \& Dong, L. (2010). Oxidative stress and DNA damage in the earthworm Eisenia fetida induced by toluene, ethylbenzene and xylene. Ecotoxicology, 19, 1551-1559.

Lowe, C. \& Butt, K. (2007). Earthworm culture, maintenance and species selection in chronic ecotoxicological studies: A critical review. European Journal of Soil Biology, 43, 281-288.

Luo, Y., Zang, Y., Zhong, Y. \& Kong, Z. (1999). Toxicological study of two novel pesticides on earthworm Eisenia fetida. Chemosphere, 39, 2347-2356.

Lyman, W., Reehl, W. \& Roselblatt, D. (1990). Handbook of chemical property estimation methods. American Chemical Society. Washington, DC.

MINAM (Ministerio del Ambiente). (2013). Aprueban Estándares de Calidad Ambiental (ECA) para Suelo. Decreto Supremo $\mathrm{N}^{\circ}$ 002-2013-MINAM. El Peruano, 25 de marzo del 2013. pp. 491497 491500 .

Morrison, D., Robertson, B. \& Alexander, M. (2000). Bioavailability to earthworms of aged DDT, DDE, DDD, and dieldrin in soil. Environmental Science \& Technology, 34, 709-713.

Murrugarra, P.F. (2009). Obtención de gasolinas de alto octano vía rectificación de una Nafta Craqueada. Tesis de Grado. Lima, Perú.

NTP (Norma Técnica Peruana). (2017). Petróleo y derivados. Gasolinas uso motor. Especificaciones. $2^{\mathrm{da}}$ Ed. Reemplaza a la NTP 321.102:2002.

OECD (Organisation for Economic Co-operation and Development). (1984). Earthworm, Acute Toxicity Tests. Guideline for Testing of Chemicals. pp. 1-9. 
Palafox, A., Hernandez, A., Lopez, J. \& Cuevas, M. (2012). Evaluación de la toxicidad de los suelos mediante bioensayos con lombrices. México, DF: Métodos Ecotoxicológicos para la evaluación de suelos contaminados con hidrocarburos.

Pasková, V., Hilscherová, K., Feldmannová, M. \& Bláha, L. (1988). Toxic effects and oxidative stress in higher plants exposed to polycyclic aromatic hydrocarbons and their $\mathrm{N}$-heterocyclic derivatives. Environmental Toxicology and Chemistry, 20, 17-23.

Patel, M., Francis, J., Cooper, E. \& FullerEspie, S. (2007). Development of a flow cytometric, non-radioactive cytotoxicity assay in Eisenia fetida. Soil Biology, 43, 97-103.

Peijnenburg, W. (2002). Bioavailability of metals to soil invertebrates. In: Allen, H.E. (ed). Bioavailability of metals in terrestrial ecosytems: Importance of partitioning for bioavailability to invertebrates, microbes and plants. Society of Environmental Toxicology and Chemistry, Pensacola, FL, pp. 89-112.

Pérez, S., Silva, I., Peñuela, G. \& Cardona, S. (2015). Evaluación de biocombustibles e hidrocarburos del petróleo (Gasolina y Diesel) en un suelo: proceso de transporte y biorremediación. Revista de la Escuela de Ingeniería de Antioquia, 2, 23-46.

PES (Parsons Engineering Science). (1999). Light nonaqueous-phase liquid weathering at various fuel relea- se sites. Consultado: http://synectics.net/public/library/StreamResource axd? DSN $=$ pub\&Mode $=F i-$ leImage_Inline $\& I D=1132$

Petroperú. (2013). Hoja de datos de Seguridad de Materiales. Obtenido de http://www.petroperu.com.pe/ portalweb/Main.asp?Seccion=55

Pinedo, A. (2014). Risk assessment applied to soils affected by petroleum hydrocarbons. España: Universidad de Cantabria. Departamento de Ingenierías Química y Biomolecular.

Pinedo, J., Ibañez, R., Lijzen, J. \& Irabien, A. (2013). Assessment of soil pollution based on total petroleum hydrocarbons and individual oil substances. Journal of Environmental Management, 130, 72-79.

Planes, E. \& Fuchs, J. (2015). Cuales son los aportes de la ecotoxicología a las regulaciones ambientales. Ciencia e Investigación, 65, 45-62.

Radamass, K., Megharaj, M., Venkateswarlu, K. \& Naidu, R. (2015). Ecological implications of motor oil poluution: earthworm survival and soil health. Soil Biology and Biochemistry, 85, 72-81.

Repsol. (2007). Ficha de datos de seguridad (conforme al DS 026-94EM). Obtenido de http://www. repsol.com/pe_es/corporacion/ complejos/refineria-la-pampilla/ conoce_refineria_pampilla/ productos_y_servicios/productos/

Salanitro, J., Dorn, P., Huesemann, M., Moore, K., Rhodes, I., Rice, L., 
Vipond, T.E., Western, M.M. \& Wisniewski, H.L. (1997). Crude oil hydrocarbon bioremediation and soil ecotoxicity assessment. Environmental Science \& Technology, 31, 1759-1776.

Sanchez-Hernandez, J.C. (2006). Earthworm biomarkers in ecological risk assessment. Reviews of Environmental Contamination and Toxicology, 188, 85-126.

Schaefer. M., Peterson. S.O. \& Filser. J. (2005). Effects of Lumbricus terrestris, Allolobophora chlorotica and Eisenia fetida on microbial community dynamics in oilcontaminated soil. Soil Biology and Biochemistry, 37, 2065-2076.

Scott-Fordsmand, J. \& Weeks, J. (2000). Biomarkers in earthworms. Review of Environmental Toxicology, 165, 117-159.

Shukry, W., Al-Hawas, G., Al-Moaikal, R. \& El-Bendary, M. (2013). Effect of petroleum crude oil on mineral nutrient elements, soil properties and bacterial biomass of the rhizosphere of jojoba. British Journal of Environment and Climate Change, 3, 103-118.

Smith, B., Greenberg, B. \& Stephenson, G. (2010). Comparison of biological and chemical measures of metal bioavailability in field soils. Chemosphere, 81, 755-766.

Song, Y., Zhou, Q., Gong, P. \& Sun, T. (2005). Ecotoxicity of soils contaminated with industrial and domestic wastewater in western
Shenyang, China. Science in China

Series C: Life Sciences, 48, 48-56.

Spurgeon, J., Weeks, J. \& Van Gestel, C. (2003). A summary of eleven years progress in earthworm ecotoxicology. Pedobiologia, 47, 558-606.

Tas, J., Balk, F., Ford, R. \& van de Plassche, E. (1997). Environmental risk assessment of musk ketone and musk xylene in the Netherlands in accordance with the EU-TGD. Chemosphere, 35, 2973-3002.

Venkateswara Rao, J., Kavitha, P. \& Pasmanabha, R.A. (2003). Comparative toxicity of tetra ethyl lead and lead oxide. Environmental Research, 92, 271-276.

Wang, W., Lyons, D., Clark, N., Gautam, M., \& Norton, P. (2000). Emissions from nine heavy trucks fueled by diesel and biodiesel blend without engine modification. Environmental Science and Technology, 34, 933-939.

Weeks, J., \& Comber, S. (2005). Ecological risk assessment of contaminated soil. Mineral Management, 69, 601-6013.

Whitfield, M., Stephenson, G.L., Simpson, A.J. \& Simpson, M.J. (2013). Comparison of earthworm responses to petroleum hydrocarbon exposure in age field contaminated soil using traditional ecotoxicity endpoint and $\mathrm{H}$ NMR-based metabolomics. Environmental Pollution, 182, 264-267. 